\title{
Passive Motor Learning: Oculomotor Adaptation in the Absence of Behavioral Errors
}

\author{
Matan Cain, Yehudit Botschko, and $\$ Mati Joshua
}

https://doi.org/10.1523/ENEURO.0232-20.2020

Edmond and Lily Safra Center for Brain Sciences, the Hebrew University, Jerusalem 91904, Israel

\begin{abstract}
Motor adaptation is commonly thought to be a trial-and-error process in which the accuracy of movement improves with repetition of behavior. We challenged this view by testing whether erroneous movements are necessary for motor adaptation. In the eye movement system, the association between movements and errors can be disentangled, since errors in the predicted stimulus trajectory can be perceived even without movements. We modified a smooth pursuit eye movement adaptation paradigm in which monkeys learn to make an eye movement that predicts an upcoming change in target direction. We trained the monkeys to fixate on a target while covertly, an additional target initially moved in one direction and then changed direction after $250 \mathrm{~ms}$. The monkeys showed a learned response to infrequent probe trials in which they were instructed to follow the moving target. Additional experiments confirmed that probing learning or residual eye movements during fixation did not drive learning. These results show that motor adaptation can be elicited in the absence of movement and provide an animal model for studying the implementation of passive motor learning. Current models assume that the interaction between movement and error signals underlies adaptive motor learning. Our results point to other mechanisms that may drive learning in the absence of movement.
\end{abstract}

Key words: adaptation; eye movements; motor learning; smooth pursuit

\section{Significance Statement}

What are the signals that drive learning? Many experimental and theoretical studies have approached this question from the perspective of motor adaptation as it is both extremely relevant to everyday life and allows for tight experimental control. Motor adaptation is thought to be a gradual process in which errors in behavior are corrected. Here, we challenged this view and developed a behavioral paradigm for studying whether movement is necessary for motor adaptation. We found that motor adaptive learning can be elicited in the absence of movement, thus suggesting that motor adaptation has a crucial passive component.

Received June 1, 2020; accepted December 22, 2020; First published February 16, 2021.

The authors declare no competing financial interests.

Author contributions: M.C. and M.J. designed research; M.C. and Y.B. performed research; M.C. and M.J. analyzed data; M.C. and M.J. wrote the paper.

This work was supported by the European Research Council (no. 755745), a Human Frontier Science Program Career Development Award (CDA00056/ 2016-C), and the Israeli Scientific Foundation (380/17).

Acknowledgements: We thank R. Raghavan for helpful comments.

Correspondence should be addressed to Matan Cain at matancain@ gmail.com.

https://doi.org/10.1523/ENEURO.0232-20.2020

Copyright (C) 2021 Cain et al.

This is an open-access article distributed under the terms of the Creative Commons Attribution 4.0 International license, which permits unrestricted use, distribution and reproduction in any medium provided that the original work is properly attributed.

\section{Introduction}

To better understand learning, the signals that drive learning need to be identified behaviorally to reveal their implementation at the neuronal level. Here, we use the characterization of motor adaptation as a gradual improvement in performance in response to altered conditions (Krakauer and Mazzoni, 2011). Motor adaptation is an especially valuable model for studying learning since experiments can reproducibly generate perturbation and then track the changes in behavior on a trial-by-trial basis. Recent research has highlighted the importance of sensory feedback on movement in driving motor adaptation. For example, the difference between the predicted and actual consequences of movement was shown to have both a computational advantage and account for behavioral results (Wolpert and Miall, 1996; Shadmehr et al., 
2010). However, feedback on movement is only one of many signals that may drive motor learning (Mazzoni and Krakauer, 2006; McDougle et al., 2016; Mostafa et al., 2019).

In terms of implementation, it has been hypothesized that in the cerebellum, movement and sensory signals converge to drive adaptive motor learning (Wolpert et al., 1998). When an erroneous motor command is executed, the climbing fiber input to the cerebellum drives plasticity that results in a more accurate upcoming movement (Gilbert and Thach, 1977; Ito, 1982; Stone and Lisberger, 1990). In the eye movement system, there is impressive trial-by-trial evidence for an association between climbing fiber input (manifested as complex spikes), the simple spike output of the Purkinje cell, and learned behavioral changes (Medina and Lisberger, 2008; Suvrathan et al., 2016; Herzfeld et al., 2018). In addition, in the eye movement system, there are extensive data showing which cerebellar sites drive eye movement and the pathways that provide signals to these areas (Voogd et al., 2012). Identifying non-motor signals in oculomotor learning can be interpreted in the context of what is already known about the implementation of motor learning and lead to testable hypothesis on where and how non-motor signals drive learning. Thus, we aimed to use an eye movement adaptation paradigm, in which a link between learning and its implementation has been establish, to test whether movement is necessary for motor adaptation.

We modified a smooth pursuit eye movement leaning paradigm to test whether sensory feedback on eye movements is needed for learning to occur. When monkeys are trained to track a moving target that repeatedly undergoes the same change in direction at a predictable time, a learned smooth pursuit eye movement is elicited before the change in target direction (Medina et al., 2005; Joshua and Lisberger, 2012). These behavioral changes occur quickly and reach near asymptotic values after 50 trials (Hall et al., 2018). During the learning of perturbed target motion, the relationship between movement and prediction target trajectory can thus be teased apart because motion can be sensed covertly without eye movement. We therefore designed a new paradigm in which monkeys learned to predict a change in direction of a target without tracking it. We termed this passive motor learning. We examined this type of learning in infrequent trials in which monkeys tracked a moving target, to show that monkeys can learn passively by observing and not tracking target motion. The interpretation of these results, together with what we already know about the pursuit system suggest testable hypotheses with respect to the areas and mechanisms involved in passive motor learning.

\section{Materials and Methods}

We collected behavioral data from two male and two female Macaca fascicularis monkeys $(4-6 \mathrm{~kg})$. All procedures were approved in advance by the Institutional Animal Care and Use Committees of the Hebrew university of Jerusalem and were in strict compliance with the National Institutes of Health Guide for the Care and Use of Laboratory Animals. We implanted head holders to restrain the monkeys' heads in the experiments. After the monkeys had recovered from surgery, they were trained to sit calmly in a primate chair (Crist Instruments) and consume liquid food rewards (infant food mixed with water and infant formula, $0.1 \mathrm{ml} /$ trial) from a tube set in front of them. We trained the monkeys to track spots of light that moved across a video monitor placed in front of them.

Visual stimuli were displayed on a monitor $65 \mathrm{~cm}$ from the monkeys' eyes. The stimuli appeared on a dark background in a dimly lit room. A computer performed all real-time operations and controlled the sequences of target motion. The position of the eye was measured with a high temporal resolution camera (1 kHz, Eye link, SR Research) and collected for further analysis. Monkeys received a reward when tracking the target successfully.

Pursuit stimuli were presented in trials. In the eye movement trials, each trial started with a circular white target that appeared in the center of the screen. After $1 \mathrm{~s}$ of presentation, in which the monkey was required to acquire fixation $\left(3 \times 3^{\circ}\right.$ window), the target began moving. The exact target trajectory is detailed below according to the different blocks. The monkeys were rewarded at the end of trials for keeping their eyes within a window of $5 \times 5^{\circ}$ around the target. We used a large fixation window so that the monkeys' behavior was not restricted during the learning trials. In the fixation trials, two targets were displayed: a stationary and a moving target. The stationary target was a $1^{\circ}$ side length square which was displayed during the entire trial. The moving target was a white circular spot (except on reward blocks, see below), similar to the target on the eye movement trials. At the beginning of each trial, the stationary target appeared in the center of the screen and the monkey was required to acquire fixation $\left(3 \times 3^{\circ}\right.$ window). After $1 \mathrm{~s}$, the moving target appeared and started to move with a trajectory that varied depending on the block. To be rewarded, the monkey had to keep its gaze on the stationary target. To keep conditions similar in the eye movement and fixation trials, we used the same size fixation window as in the eye movement trials. We verified the potential confound that the monkeys might initially track the moving target although they were instructed to fixate. We confirmed that the monkeys only made very small eye movements during the fixation trials and we designed experiments to control for this movement (see below, paradigm 3). Trials were considered to have failed if the monkey interrupted fixation at any step during the trial. After a failed trial, the same trial was presented to the monkey until success.

The paradigms consisted of learning blocks interleaved with washout blocks (if not specified otherwise). Each block consisted of 100 successful trials. We detail the composition of the different learning blocks below. Washout blocks consisted of 50 eye movement trials and 50 fixation trials interleaved randomly in which after $1 \mathrm{~s}$, the moving target stepped to a $4^{\circ}$ eccentric position and started to move in the opposite direction at $20 \%$ s (stepramp; Rashbass and Westheimer, 1961). The target continued to move for $650 \mathrm{~ms}$ after motion onset and then stopped and stayed still for an additional $500 \mathrm{~ms}$. 


\section{Paradigm 1: motor blocks, fixation congruent, and incongruent blocks}

The motor blocks consisted of 100 eye movement trials in which the target moved initially in one direction and then after $250 \mathrm{~ms}$, an orthogonal $20 \% \mathrm{~s}$ component of motion was added (Medina et al., 2005). We term the direction of the initial target motion and the direction of the orthogonal component the base and learned directions. To select the learned direction, we prescreened the monkeys' behavior to select target motion directions in which we could consistently drive learning. Specifically, these directions consisted of down and rightward for the base and learned directions.

The fixation congruent and incongruent blocks consisted of 90 fixation trials and 10 eye movement trials. In the fixation trials, in the congruent blocks, the moving target changed direction (the same as for trials in the motor learning block). In fixation trials in the incongruent blocks the moving target did not change direction (similar to trials in the washout blocks). In the eye movement trials in both fixation blocks (congruent and incongruent) the target changed direction (same as in trials in the motor learning blocks). In both types of fixation blocks, each group of 10 trials included nine fixation trials and one eye movement trial introduced randomly between them. Motor, congruent and incongruent blocks were randomly interleaved and separated by washout blocks. The average learned response at the end of the washout blocks (25 last eye movement trials) was defined as the baseline level. We recorded this paradigm for $7 \mathrm{~d}$ for each monkey which typically consisted of nine learning blocks (three of each type) and nine washout blocks.

\section{Paradigm 2: fixation blocks without change in direction in eye movement trials}

In this paradigm, we compared two types of fixation learning blocks. The first learning block consisted of $90 \%$ of fixation trials in which the moving target changed direction. In the following learning block the learning direction was rotated $180^{\circ}$. In both blocks, in eye movement trials $(10 \%)$, the target did not change direction (same as for trials in the washout blocks). We recorded this paradigm for $3 \mathrm{~d}$ for each monkey which typically consisted of 24 learning blocks (12 of each type). In this paradigm, we directly compared adjacent blocks with opposite learning direction; therefore, we did not need to introduce washout blocks to assess learning.

\section{Paradigm 3: small angle and no angle blocks}

In this paradigm, we compared two blocks that only included eye movement trials. In the no angle blocks, in most trials $(90 \%)$, the target did not change direction (same as for trials in the washout blocks). In the small angle blocks, in most trials $(90 \%)$ the target changed direction $250 \mathrm{~ms}$ after motion onset (as in the motor blocks) but the velocity component in the learned direction was only $0.5 \%$ s. In the two blocks, learning was assessed using eye movement trials (10\%) in which a $20 \%$ s orthogonal component was added after $250 \mathrm{~ms}$ of target motion (same trials as in the motor blocks). We recorded this paradigm for $3 \mathrm{~d}$ for each monkey which typically consisted of 12 learning blocks (six of each type) and 12 washout blocks.

\section{Paradigm 4: motion and position blocks}

In this paradigm, we compared two learning blocks, the motion blocks were similar to the fixation congruent blocks in which during fixation (90\%) and the movement trials $(10 \%)$ the moving target changed direction. In the position blocks during the fixation trials $(90 \%)$, the moving target vanished at the change in direction $(250 \mathrm{~ms}$ after motion onset) and reappeared at the end of motion (650 ms after motion onset). The remaining 10\% were eye movement trials in which the target changed direction. We recorded this paradigm for $3 \mathrm{~d}$ for each monkey which typically consisted of 12 learning blocks (six of each type) and 12 washout blocks.

\section{Paradigm 5: congruent rewarded block and incongruent rewarded block}

In this paradigm, we compared two learning blocks with two types of fixation trials ( $45 \%$ each) and eye movement trials (10\%). In both blocks, the moving target changed direction in half of the fixation trials and did not change direction in the remaining trials. In the congruent rewarded blocks, the monkey was only rewarded when the moving target changed direction. In the incongruent rewarded blocks, the monkey was only rewarded when the moving target did not change direction. The color of the moving target signaled the presence of reward. In the rewarded fixation trials, a green moving target was used for Monkey $\mathrm{C}$ (blue for Monkey A) and an orange target for non-rewarded trials (pink for Monkey A). Monkeys were familiar with the color-reward association as we used the same monkeys with the same associations in prior studies (Larry et al., 2019; Lixenberg et al., 2020). The eye movement trials $(10 \%)$ were identical to those described in the motor blocks (with a regular white target). We recorded this paradigm for $3 \mathrm{~d}$ for each monkey which typically consisted of 12 learning blocks (six of each type) and 12 washout blocks

\section{Paradigm 6: learning with multiple base and learned directions}

In this part of the experiment, we compared fixation congruent and motor blocks when we interleaved blocks with many base $\left(0^{\circ}, 90^{\circ}, 180^{\circ}\right.$, or $\left.270^{\circ}\right)$ and learned (clockwise and counter clockwise) directions. Blocks were selected pseudorandomly such that all the directions had to be selected once before any direction was selected another time. Learning blocks were interleaved with washout blocks where the base direction was similar to the base direction in the subsequent learning block. We recorded data for $8 \mathrm{~d}$ for each monkey, which resulted in four motor and fixation blocks in each direction.

Paradigms 1-5 were administered to two monkeys (A and $\mathrm{C}$ ), whereas paradigm 6 was administered to the other two monkeys ( $\mathrm{E}$ and $\mathrm{F}$ ). 

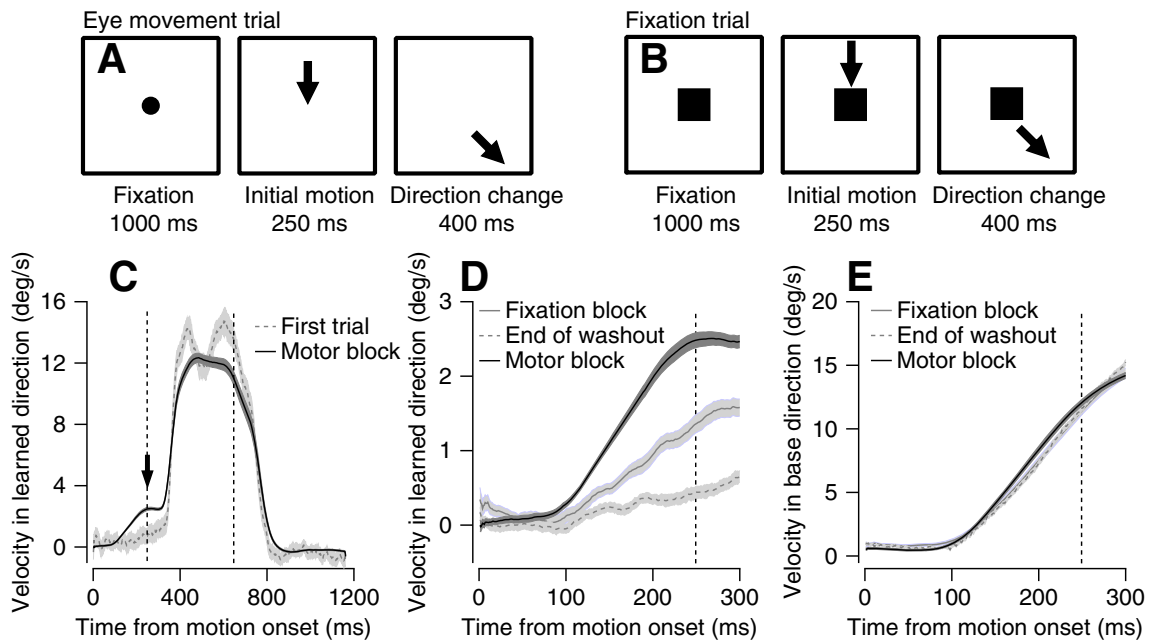

Figure 1. Trial schematics and behavior in motor and fixation blocks. $\boldsymbol{A}, \boldsymbol{B}$, Schematics of the eye movement $(\boldsymbol{A})$ and fixation $(\boldsymbol{B})$ trials. Arrows show the direction of target motion, circle represents the target before motion onset, and squares represent the fixation target. $\boldsymbol{C}$, Average eye movement in the learned direction on the first trial of learning (dashed gray trace) and postlearning trials $\left(50^{\text {th }}\right.$ to $100^{\text {th }}$ trial) averaged across all motor blocks (black). $\boldsymbol{D}, \boldsymbol{E}$, Average eye movement in the learned $(\boldsymbol{D})$ and base $(\boldsymbol{E})$ directions at the end of washout blocks (25 last eye movement trials, dashed gray) and after learning on motor blocks $\left(50^{\text {th }}\right.$ to $100^{\text {th }}$ trial averaged across all motor blocks, solid black) and fixation blocks (5th to 10th eye movement trials averaged across all congruent fixation blocks, solid gray). In all traces, shadowing represents SEM. Vertical dashed lines show the time of the change of direction (250 ms) and end of target motion (650 ms).

\section{Data analysis}

Learned velocity was computed as the velocity in the learned direction minus the average eye velocity of the last 25 eye movement trials in the corresponding washout blocks. The learned response was computed as the average learned velocity during the $100 \mathrm{~ms}$ around the change in direction in eye movement trials. We adjusted the signs of the data such that positive values of learning indicate eye velocity in the learning direction. We estimated the growth of learning $(L)$ over trials by fitting the sum of two exponentials to the learned responses.

$$
L=A_{1}\left(1-e^{\left(-T / \tau_{1}\right)}\right)+A_{2}\left(1-e^{\left(-T / \tau_{2}\right)}\right)+c,
$$

where $A_{x}$ is the peak magnitude of learning, $\tau_{x}$ is the "time constant" of learning, $T$ is the trial number -1 and $c$ is the baseline.

We used eye velocity and acceleration thresholds to detect saccades automatically and then verified the automatic detection by visual inspection of the traces. The velocity and acceleration signals were obtained by digitally differentiating the position signal after we smoothed it with a Gaussian filter with a standard deviation of $5 \mathrm{~ms}$. Saccades were defined as an eye acceleration exceeding $1000 \% \mathrm{~s}^{2}$, an eye velocity crossing $15 \%$ s during fixation or eye velocity crossing $50 \%$ shile the target moved. We first removed the saccades and treated them as missing data. We then averaged the traces with respect to the target motion onset. Finally, we smoothed the traces using a moving average filter with a span of $21 \mathrm{~ms}$.

To calculate the ratio between the learned response in the motor to other blocks, we first computed the averaged learned response across monkeys and trials in eye movement trials in the motor, congruent fixation and incongruent fixation blocks. Then, we divided the average learned response of the corresponding block by the average learned response in the motor blocks.

\section{Results}

\section{Learning to predict changes in target direction by observation}

We used a smooth pursuit eye movement learning paradigm in the monkeys (Fig. 1A,B), to test whether feedback on behavioral errors was needed to adjust behavior. The first step consisted of a motor learning block (Medina et al., 2005; Joshua and Lisberger, 2012), where the monkeys tracked a single moving target that changed direction $250 \mathrm{~ms}$ after the onset of motion (Fig. 1A, eye movement trial). We term the direction in which the target initially moved the base direction (downward in Fig. 1A) and the orthogonal direction in which we later added a velocity component the learned direction (rightward in Fig. $1 A)$. In the initial learning trials, the eye movement in the learned direction was reactive rather than predictive. After the target changed direction, the eye moved abruptly with a visually driven characteristic reaction time $(\sim 100 \mathrm{~ms}$; Fig. $1 C$, gray line). After several repetitions of trials with a change in direction, the monkeys learned to predict the upcoming motion and moved their eyes in the learned direction even before the target changed direction (Fig. 1C, black line, arrow points to the learned component). In this paradigm, the predictive eye velocity was not sufficient to completely match the upcoming target motion, so that the monkeys still abruptly responded to the change in direction (Fig. 1C, black line), which was often followed by a catchup saccade (data not shown). To avoid confounding 
the learned with the visually driven response, the analysis here was restricted to the first $300 \mathrm{~ms}$ after motion onset in the base direction (Fig. 1D,E).

Theories of motor learning often assume that sensory feedback on movement errors in learning trials drives subsequent learning (Ito, 1972; Ito and Kano, 1982; Wolpert, 1998). To test whether the feedback on eye movement is necessary for learning, we designed an additional learning block, termed the fixation block, in which the target changed direction, but the monkey did not follow it. In most trials (90\%), the monkeys were required to maintain fixation on a square in the center of the screen while the moving target changed direction (Fig. 1B). Unlike the eye movement trials, in the fixation trials, the monkeys were passive: they fixated the center of the screen, which prevented them from tracking the moving target and responding to the change in motion direction.

We tested learning in a small fraction of trials (10\%) in which the square fixation target was not displayed, and the monkeys were required to follow the moving target exactly as in the eye movement trials (Fig. 1A). In these trials, the monkeys shifted their gaze in the direction of motion even before the target changed direction (Fig. 1D, gray solid trace). To assess whether the monkeys indeed learned from these fixation trials, we compared the learned response in the fixation blocks to the end of the washout blocks. The washout blocks consisted of 100 trials in which the target never changed direction (see Materials and Methods). By the end of the washout block (termed baseline trials), the eye velocity in the learned direction was close to zero (Fig. 1D, dashed trace). We quantified the learned response as the average eye velocity in the learned direction between 200 and 300 ms after motion onset. The learned response was maximal for the motor learning blocks, intermediate in the fixation blocks, and the smallest in the washout blocks (Friedman test, $p$ $=10^{-12}$, post hoc signed-rank test with Bonferroni correction, motor $>$ fixation $p=1.2 \times 10^{-9}$, fixation $>$ washout, $\left.p=2.5 \times 10^{-9}, n=46\right)$. As expected, there were only very minor difference between these three conditions in the base direction (Fig. 1E), indicating that the learned response indeed reflected a change in eye movement direction and not an overall gain (Hall et al., 2018). Thus, in sessions with infrequent eye movement trials, the monkeys adjusted their behavior to the change in target motion, suggesting that learning was acquired in fixation trials without movement.

\section{Movement in infrequent trials does not explain the learned response in fixation blocks}

Next, we ruled out the possibility that learning in fixation blocks was driven solely by the infrequent trials (10\%) in which the monkeys tracked the target. We tested the behavior of the monkeys in additional learning blocks in which the target did not change direction on the fixation trials (Fig. 2A). We termed these blocks incongruent learning blocks (Fig. $2 A$, right) and the blocks in which the target changed direction in fixation trials as it did in the movement trials congruent learning blocks (Fig. 2A, middle). The learned response in the fixation incongruent
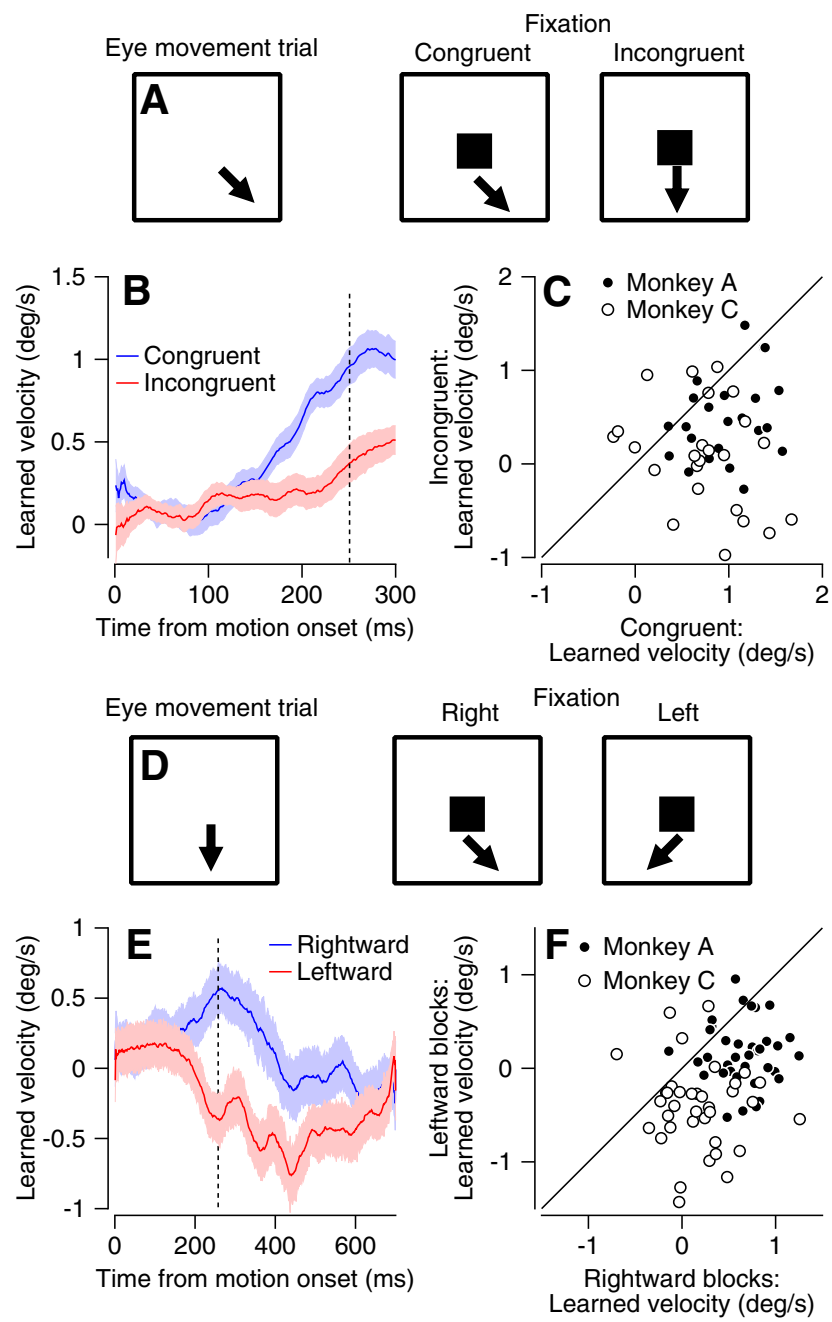

Figure 2. Learning from observation is not driven solely by infrequent eye movement trials. $\boldsymbol{A}$, Schematics represent the direction change epoch in the different experimental conditions. Left, Eye movement trials with change in direction. Middle, Congruent trial-fixation trial with directional change. Right, Incongruent trial-fixation trial without directional change. $\boldsymbol{B}$, Average learned eye velocity as a function of time from motion onset for eye movement trials averaged across all congruent (blue) and incongruent (red) blocks. $\boldsymbol{C}$, Learned response on incongruent (vertical) versus congruent (horizontal) blocks. Filled and open symbols show data from Monkeys A and C. Solid line indicates unity. $\boldsymbol{D}$, Schematics represent the target motion in the different experimental conditions. Left, Eye movement trials without a change in direction. Middle, Fixation trials in which rightward is the learned direction. Right, Fixation trial in which leftward is the learned direction. $\boldsymbol{E}$, Average learned eye velocity in eye movement trials averaged across all learning blocks as a function of time from motion onset in blocks in which the moving target moved rightward (blue) or leftward (red). $\boldsymbol{F}$, Learned response in adjacent blocks in which on fixation trials the target moved rightward (horizontal) or leftward (vertical). Filled and open symbols show data from Monkeys A and C. Solid line indicates unity. In all traces, shadowing represents the SEM. Vertical dashed line shows the time of the change in direction of the moving target. 
learning blocks could only result from the repetition of the eye movement trials. Thus, if learning were driven solely by infrequent eye movement trials, we would expect that the learned response would be similar on the congruent and incongruent blocks. When tested on the infrequent $(10 \%)$ eye movement trials, the eye velocity in the learned direction was lower in the incongruent than in the congruent learning blocks (Fig. 2B). Paired comparisons between nearby congruent and incongruent blocks that were recorded the same day (but separated by at least one washout block; see Materials and Methods) indicated that in most sessions, the learned response was higher in congruent blocks than in incongruent blocks (Fig. 2C, signed-rank test $\left.p=5.9 \times 10^{-6}\right)$. These results indicate that fixation trials play an important role in the development of the learned response.

This conclusion draws on the assumption that the contribution of the eye movement trials to the learned response was identical in the fixation congruent and incongruent blocks. To further confirm that the monkeys indeed learned from the congruent fixation trials, we tested additional learning blocks. As in the fixation congruent trials, the target changed direction in the fixation trials, but unlike the previous learning blocks we probed learning using trials in which the target did not change direction (these trials were thus identical to the eye movement trials in the washout blocks, see Materials and Methods; Fig. 2D, left). The only signal that could be used for learning in these blocks was the change in direction in the fixation trial. We alternated blocks in which the fixation trials had opposite learned directions, i.e., left or right (Fig. $2 D$ ). Thus, this experimental design had the advantage that in each learning block the monkeys never followed a target moving in the learned direction in the eye movement trials and that on the fixation trials, the target always changed direction.

In the eye movement trials, the average eye velocity deflected toward the learned direction (Fig. 2E). Positive and negative values in this analysis indicate movement right and left. Importantly, this deflection was not visually driven because the stimulus in eye movement trials did not have any motion in the learned direction. Therefore, this deflection could only have resulted from learning in fixation trials. To directly compare sessions, we plotted the learned component in alternating blocks with the opposite learned directions. The bias in the learned response toward the change in direction was manifested by the strong tendency of the dots to plot beneath the equality line in Figure $2 F$ (signed-rank test, $p=7.7 \times 10^{-10}$ ). We found a slight difference between monkeys. In Monkey $C$ the bias was symmetric, i.e., in each learning block the eye moved toward the direction of the change in target motion (positive and negative horizontal and vertical values; Fig. 2F, open dots). The movement of Monkey A was slightly biased toward positive values (corresponding to motion to the right), as indicated by the positive values on the horizontal axis and close to 0 on the vertical axis shown by the open dots in Figure 2F. Nevertheless, the comparison between blocks indicated that in both monkeys the change in direction on the fixation trials biased the learned eye velocity in the corresponding direction. Thus, the monkeys learned passively, when the only signal for learning was the change in target direction on the fixation trials.

\section{Control for movements in the fixation window}

So far, we have shown that monkeys learn from fixation trials, suggesting that neither the corrective movement nor the feedback on erroneous behavior was necessary for learning. One possible confounding effect is that monkeys did not completely suppress behavior on the fixation trials (Fig. 3B,C, solid traces). To control for this eventuality, we conducted experiments to confirm that the behavioral responses on the fixation trials did not affect the learned response.

In the learned direction on the fixation trials, we observed a very slight increase in the velocity around the change in target direction in the congruent blocks compared with the incongruent blocks (Fig. 3C, arrow marking solid blue and red traces, Fig. $3 D$, gray trace). We aimed to mimic this behavioral difference to test whether it would impact the learned response on motor trials. To mimic the visually driven eye movement in the learned direction on congruent trials, the monkeys were required on most trials (90\%) to track a moving target that changed direction slightly after $250 \mathrm{~ms}$ such that a small component $(0.5 \%$ s) of the target velocity was added in the learned direction (Fig. $3 A$, bottom left). In the second block, which was designed to mimic behavior on incongruent trials, in $90 \%$ of the trials the target did not change direction (as in the eye movement trials in the washout blocks, see Materials and Methods; Fig. $3 A$, bottom right). As expected, the difference in eye velocity in the learning direction between learning trials consisting of no angle and small angle blocks (Fig. $3 D$, black) was indeed similar to the difference between fixations trials in the congruent and incongruent blocks (Fig. 3D, gray). To keep the structures of the blocks as similar as possible and to probe learning, in the remaining $10 \%$ of the trials, the target changed direction as in the previous experiments $(20 \% \mathrm{~s}$ component in the learning direction; Fig. $3 A$, top).

If indeed the corrective behavior we observed on the fixation trials were sufficient to drive learning, we would expect to find a difference between the mimic blocks with and without the small angle. However, we found that the difference between the learned response eye velocity on blocks with small and no angle was not significant (Fig. $3 E$, Wilcoxon signed-rank test, $p=0.26$ ). Furthermore, the difference between the learned response in the congruent versus incongruent blocks was larger than the difference between blocks with and without an angle (rank-sum $p=0.036$ ). Therefore, this control suggests that the slight corrective movement we observed in the fixation trials did not drive learning.

Next, we focused on the increase in base velocity on fixation trials around the change in direction in both the congruent and incongruent blocks (Fig. 3B, solid blue and red traces, marked by an arrow). This movement might contribute to learning since the discrepancy between the movement and the direction of target change could elicit 

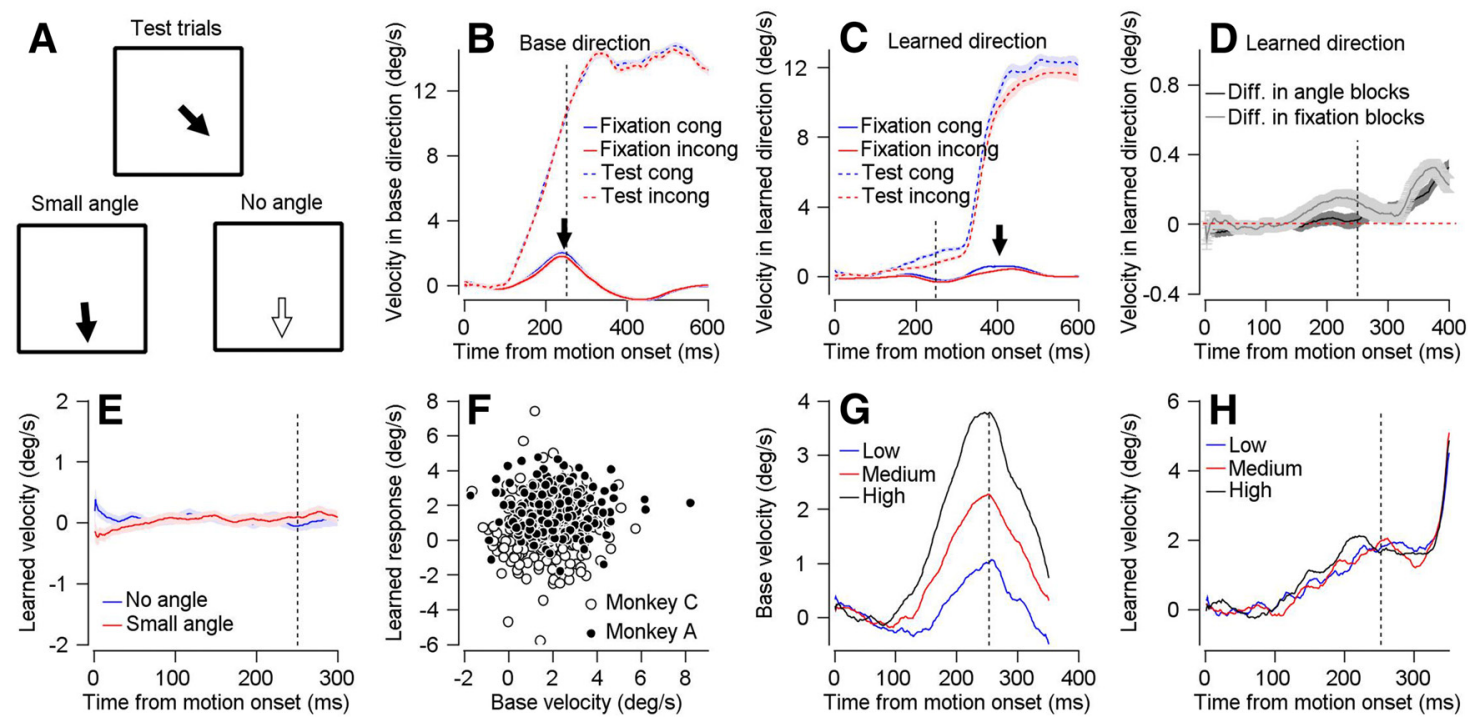

Figure 3. Learning is not driven by residual movement on fixation trials. $\boldsymbol{A}$, Schematics showing the direction of motion change on trials with large (top), small (bottom left) and no change (bottom right) in target direction. $\boldsymbol{B}, \boldsymbol{C}$, Eye velocity in base (B) and learned $(\boldsymbol{C})$ direction as a function of time from motion onset on fixation (solid trace) and test (dashed trace) trials. Blue and red traces show the velocity averaged across congruent and incongruent fixation blocks. $\boldsymbol{D}$, Difference in learned eye velocity between fixation trials from congruent and incongruent blocks (gray) and difference between trials with small and no angle in the corresponding blocks (black). Dashed red line indicates null velocity. $\boldsymbol{E}$, Average learned eye velocity as a function of time from motion onset in test trials in blocks without change in direction (blue) and with a small change in direction (red). $\boldsymbol{F}$. Base velocity on fixation trials, average from 200 up to 300 ms after motion onset versus learned response in subsequent test trials in fixation congruent blocks. Filled and open symbols show data from Monkeys $A$ and C. $\boldsymbol{G}, \boldsymbol{H}$, Base velocity on fixation trials $(\boldsymbol{G})$ and learned velocity on eye movement trials $(\boldsymbol{H})$ in fixation congruent blocks as a function of time from motion onset for group of fixation trials with low, medium, and high base velocities (blue, red, and black traces). In all traces, shadowing represents the SEM. Vertical dashed line shows the time of the change in direction of the moving target.

an error signal. However, if indeed this discrepancy between behavior and target motion drove learning, we would expect that larger movements in the base direction would correlate with more learning on the movement trials. However, in the congruent blocks, there was no significant correlation between the base velocity averaged across the fixation trials and the amplitude of the learned response on the subsequent test trial (Fig. 3F, the multiple regression analysis with monkeys and base velocity as predictors of learned velocity was significant for monkeys, $p=3.02 \times$ $10^{-13}$, but not for base velocity, $p=0.34$ ). Figure $3 G, H$ shows the absence of correlation in time for Monkey $A$. We clustered the base velocity on the fixation trials into three groups according to the magnitude of the base direction eye velocity on the fixation trials (Fig. 3G). As expected from a non-correlated relationship, these clusters were not preserved when we plotted the learned response on the eye movement trials (Fig. $3 H$ ). These result are consistent with a recent study using a motor learning paradigm which did not find a correlation between movement speed in the base direction before change in the target direction and learning on the next trial (Herzfeld et al., 2020). Thus, it is unlikely that residual movement on the fixation trials within the fixation window was necessary for learning.

\section{Learning in fixation blocks is driven by the change in direction}

We have shown that the monkeys were able to learn from fixation trials. We next attempted to better understand which component in the fixation trials was necessary for learning. In the eye movement trials, the crucial instructive signal for learning is the change in target direction (Medina and Lisberger, 2008; Yang and Lisberger, 2014). Consequently, we tested whether motion in the learned direction of the target is essential to develop the learned response. Alternatively, information about the end point position of the target could be sufficient to drive learning. To answer this question, we compared the learned response in two learning blocks. The first block was identical to the fixation congruent block described above (Fig. 4A, top and middle). In this context we termed this block the motion block. The second block, termed the position block was similar to the previous block except that the moving target vanished right before the addition of the upward velocity component, $250 \mathrm{~ms}$ after motion onset. The target then reappeared at the end of the trial (650 ms after motion onset) in the same position as in the motion trials (Fig. 4A, bottom). We found that the learned response on the motion block was higher than on the position block (Fig. 4B). Single-session comparisons indicated that this difference was significant (Fig. 4C, Wilcoxon signed-rank test, $p=1.8 \times 10^{-4}$ ), consistent across monkeys and observed in most sessions. Therefore, instructing learning without target motion was less effective in driving passive learning. This result highlights the important role of motion in the development of the learned response (for possible interpretations, see Discussion). 
A
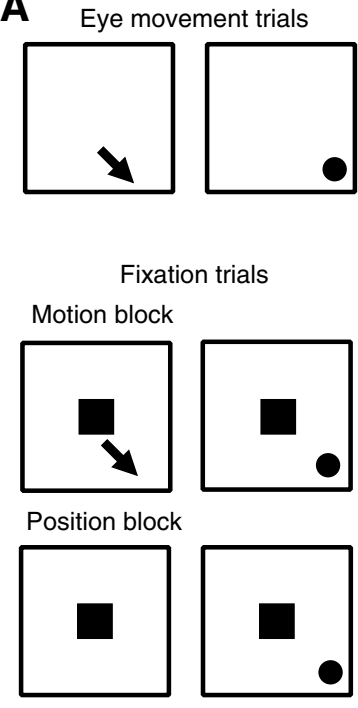
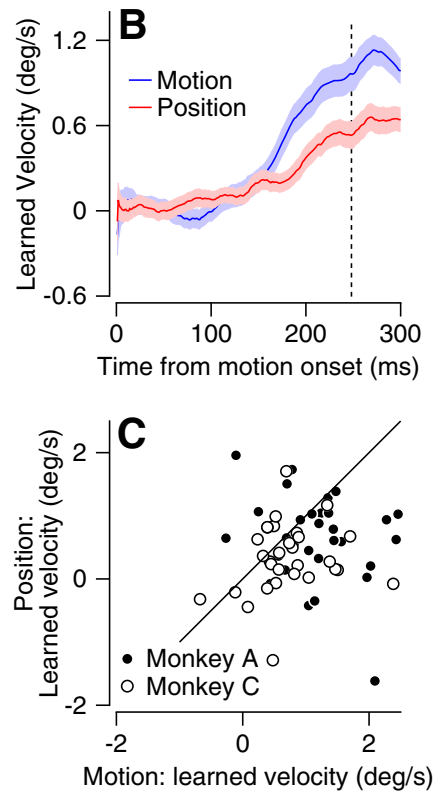

\section{Learned response in fixation blocks is modulated by expected reward}

We have shown how basic sensorimotor parameters such as target motion and eye movements impact learning. We next tested whether the task's broader context could also influence learning from observation. Specifically, reward interacts with the visuomotor processing of the pursuit system (Joshua and Lisberger, 2012; Damasse et al., 2018; Lixenberg and Joshua, 2018). We therefore designed a task to test whether the learned response could be modulated by reward information. The structure of the eye movement and fixation trials were similar to those described in the first part of the experiment (Fig. 1A). Each block consisted of $10 \%$ eye movement trials and $90 \%$ fixation trials. The fixation trials were equally divided (45\%) into congruent trials and incongruent trials. The key difference was that the reward associated with each fixation trial was swapped between blocks. In the congruent-reward blocks, a reward was only given after congruent trials (Fig. $5 A$, top), whereas in the incongruent-reward blocks, a reward was only given after incongruent trials (Fig. 5A, bottom). The color of the target indicated whether the monkey would be rewarded at the end of the trial (Fig. $5 A$ ). We tested learning in eye movement trials with a white target in which the monkey always received a reward, to ensure that the reward in these trials did not affect the expression of learning differently (Joshua and Lisberger, 2012).

We found that reward modulated the amplitude of the learned response. The average learned response on the eye movement trials was higher for the congruent-reward than for the incongruent-reward blocks (Fig. 5B). Paired tests between interleaved blocks that were separated by a washout block indicated this difference was significant $\left(p=6.1 \times 10^{-5}\right.$, signed-rank test; Fig. $\left.5 C\right)$. These results corroborate the hypothesis that reward modulation affects the acquisition of learning as was found in some paradigms of motor learning (Liu et al., 2019) but not in others (Joshua and Lisberger, 2012). Here, we aimed to optimize the conditions for finding an effect of reward on
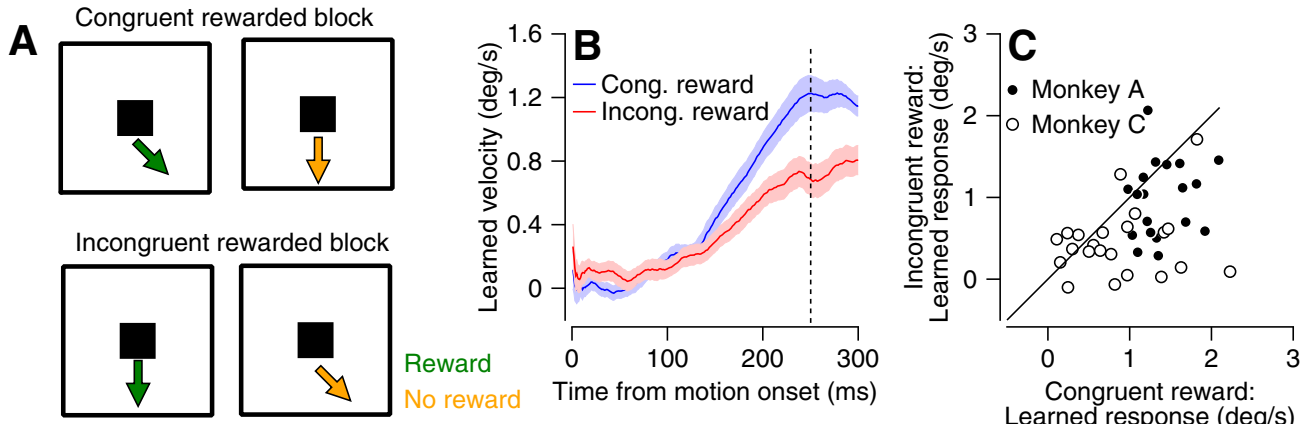

Figure 5. Learning on fixation blocks is modulated by expected reward. $\boldsymbol{A}$, top, Fixation trials in congruent rewarded blocks. Left, Congruent rewarded trials. Right, Incongruent unrewarded trials. Bottom, Fixation trials in incongruent rewarded blocks. Left, Incongruent rewarded trials. Right, Congruent unrewarded trials. Colors correspond to the color of the target used for Monkey $\mathrm{C}$. For Monkey A blue and pink signaled reward and omission of reward. B, Learned eye velocity as a function of time from motion onset averaged across all eye movement trials in congruent rewarded (blue) and incongruent rewarded (red) blocks. $\boldsymbol{C}$, Learned response in congruent rewarded (horizontal) versus incongruent rewarded (vertical) blocks. Solid line indicates unity. One outlier that had values of $(0.52 ;-1.53)$ is not shown. Filled and open symbols show data from Monkeys $A$ and $C$. In all traces, shadowing represents the SEM. Vertical dashed line shows the time of the change in direction of the moving target. 

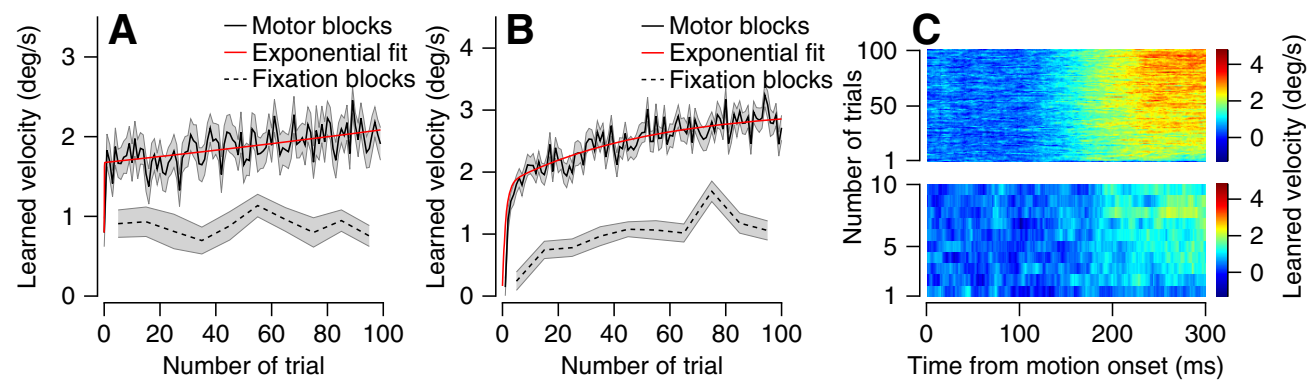

Figure 6. Learning dynamics in motor and fixation congruent blocks. $\boldsymbol{A}$, $\boldsymbol{B}$, Learning curve for motor (solid) and congruent fixation blocks (dashed) with single $(\boldsymbol{A})$ or multiple $(\boldsymbol{B})$ base and learned directions. Exponential fit of the motor learning curve is shown in red. In all traces, shadowing represents the SEM. C, Learned velocity in eye movement trials averaged across all motor (up) and fixation blocks (bottom). Colors represent learned velocity, and each horizontal line of the image shows eye velocity as a function of time for a single trial. The trials in a learning block progress from the bottom to the top of the image. The left plot shows data from Monkeys $\mathrm{A}$ and $\mathrm{C}$; the middle and right from Monkeys $\mathrm{E}$ and $\mathrm{F}$.

passive learning by making the experimental conditions similar to experiments that have demonstrated that reward affects the acquisition of motor learning (Liu et al., 2019). Therefore, we interleaved trials with different reward outcomes and different effects on learning (incongruent/congruent). To compare the effects of reward on motor and passive learning, a better characterization of the condition in which reward drives the acquisition of motor learning is needed. This characterization is important but beyond the scope of the current study. Note that before the experiment, the monkeys were extensively trained to associate color with the reward (Larry et al., 2019; Lixenberg et al., 2020). Therefore, it is likely that the expected reward, rather than reward delivery, was the critical reward signal modulating learning, perhaps through attention mechanisms.

\section{Very rapid learning is probably explained by the uniformity of the learning block}

In the previous sections, we considered learning blocks as a whole without addressing the dynamics of learning. We calculated the learning curve in the fixation and motor blocks by assessing the size of the learned response as a function of the number of trials (Fig. 6A). In the fixation blocks, we did not observe a progression in learning (Fig. $6 A$, dashed line), indicating that most of the learning occurred before the first eye movement trial. In the fixation blocks, the learned response on the first eye movement trial (which was followed on average by five fixation trials) was not significantly different from the other eye movement trials $(p=0.8$, rank-sum test). To test whether this quick learning was specific to the fixation block, we analyzed the learning curve in the interleaved motor learning blocks. We found that as in the fixation block, most of the learning occurred very rapidly (Fig. $6 \mathrm{~A}$, solid). To quantify, we fit the learning curve to a double exponent (see Materials and Methods). We found that the rapid learning $\left(\tau_{1}=4 \times 10^{-2}\right.$ trials) dominated the learning process in that it explained $68.46 \%$ of the learning in the first 100 trials, suggesting that the absence of graduality in passive learning was because of the high speed of learning.

The main goal of this study was to test whether monkeys can learn without tracking the target. We therefore attempted to strictly control parameters such as the direction of motion that a-priori seemed irrelevant. However, this choice might have led to the very fast learning in motor blocks and our inability to detect changes in fixation blocks (Fig. 6A). To test whether indeed restricting the direction led to the fast learning, and to test for dynamics in the passive learning, we conducted an experiment in which we enriched the context by varying the base $\left(0^{\circ}, 90^{\circ}, 180^{\circ}\right.$, or $\left.270^{\circ}\right)$ and learned (clockwise and counter clockwise) directions of the fixation congruent and motor blocks (on two other monkeys). The learning curve in this richer context increased gradually in both the fixation and motor learning blocks (Fig. $6 B, C)$. In the motor blocks rapid learning dropped to $56 \%$ of the total learning and was slower than in the homogeneous context $\left(\tau_{1^{\prime}}=1.2\right.$ trials). Thus, the richness of the direction influences the learning dynamics as do other task parameters such as the time between consecutive learning trials or different trials interleaved between learning trials.

\section{Discussion}

\section{Passive motor learning}

It is well established that monkeys learn to predict a change in target direction when actively tracking the target (Medina et al., 2005). Here, we found that a passive observation of the change in target direction without tracking is sufficient to elicit a learned response. Thus, an association between motor output and sensory feedback is not necessary to elicit an adaptive response. Other studies on adaptation paradigms have highlighted the importance of sensory feedback on movement in learning (Held and Freedman, 1963; Mazzoni and Krakauer, 2006; Mostafa et al., 2019). All these paradigms have reported a discrepancy between the predicted and observed sensory outcomes of motor commands (Shadmehr et al., 2010). For example, application of a force field is known to change the observed sensory outcomes of a given motor command. The smooth pursuit paradigm presented here differs from these paradigms in that the perturbation (the change in direction of the moving target) can be perceived without movement so that learning does not depend on the predicted sensory outcomes of a motor command. This difference may explain why the 
pursuit system could be more amenable to passive motor learning.

Nevertheless, it remains unclear which signals drive passive learning. There are at least two possible mechanisms governing the ways in which velocity signals in fixation trials could drive learning. The first is that velocity is an arbitrary cue associated with the direction of movement on eye movement trials. This type of cue might be used as a signal for switching movement in the subsequent eye movement trial according to the direction of the moving target in the fixation trials. The second possible mechanism is that learning acts specifically on the velocity signals. The position experiment (Fig. 4) lends more weight to the latter alternative since it showed that another relevant cue, the position of the target at the end of the movement trials, drove less learning, thus suggesting that passive learning is not exclusively underpinned by switching the movement between blocks. Additional evidence for the importance of velocity signals beyond arbitrary rules comes from pursuit motor learning in which target motion direction rather than abstract rules such as alternation of the learned direction drive motor learning (Yang and Lisberger, 2010). Thus, it is probable that in the pursuit learning, velocity signals play a unique role. However, we cannot completely refute the possibility that the velocity, as a very salient signal, was easier for the monkeys to interpret as a cue.

Overall, passive (as well as motor) learning in smooth pursuit in monkeys is probably mostly implemented through the sensorimotor representation of the target motion rather than an abstract representation. The smooth pursuit eye movement system has been widely used as a model system for studying sensorimotor transformation and motor learning at the implementation level of neurons and networks (Lisberger, 2010; Joshua and Lisberger, 2015). The paradigm we developed here can be harnessed to provide testable hypotheses on where and how the brain implements passive learning. Another advantage of this paradigm stems from the temporal gap between the sensory inputs on the fixation trials and their effect on later motor trials. Thus, this paradigm provides an easy way to dissociate between the processing of visual motion and the generation of pursuit motor commands for the upcoming movement.

\section{Possible neural implementation in the cerebellum and frontal cortex}

The cerebellar flocculus plays an important role in the development of a predictive response to an instructive change in target direction during active motor learning (Medina and Lisberger, 2008). According to the classic cerebellar model, sensory errors resulting from inaccurate movement drive climbing fiber input (Albus, 1971; Ito, 1972; Gilbert and Thach, 1977). The climbing fiber input, paired with input to the Purkinje cell, results in an associative reduction in synaptic strength (Ito et al., 1982; Suvrathan et al., 2016). This reduction is thought to underlie the subsequent improvement in behavior.

Tracking is not necessary for climbing fiber activation, as a task-irrelevant background motion was shown to have a substantial effect on the climbing fiber response
(Guo et al., 2014). Similarly, motion of the background in fixation trials (i.e., the moving target), may drive climbing fiber input as well. The error signal in this framework might be the predicted motion of the target relative to the actual moving target trajectory. In addition, to elicit behavioral learning, climbing fiber activation must be coupled with the appropriate parallel fiber input. It is possible that the appropriate parallel fibers are also activated during fixation trials since some of the activity of the Purkinje cell is driven by sensory responses (Krauzlis and Lisberger, 1991) or might reflect a motor command that is cancelled downstream. According to this hypothesis, the same cerebellar mechanisms would drive active and passive learning. At the neuronal level, it predicts that all the hallmarks of cerebellar learning will be observed during passive learning. For example, in fixation trials, climbing fiber inputs will be modulated during the target change of direction. Furthermore, the Purkinje cell simple spikes are likely to be tightly related to the climbing fiber input on a trial-by-trial basis (Medina and Lisberger, 2008; Suvrathan et al., 2016; Herzfeld et al., 2018). The presence of a climbing fiber response after the change in direction on one trial should be associated with a change in the simplespike firing rate on the subsequent fixation or eye movement trial.

Passive learning could be implemented in the frontal eye field (FEF). Visual, motor and temporal signals converge in the FEF (Bruce and Goldberg, 1985; Macavoy et al., 1991; Schall et al., 1995; Sommer and Wurtz, 2006; Schoppik et al., 2008; Schafer and Moore, 2011). In the classic active smooth pursuit paradigm with change in direction, neurons that are temporally tuned to the time of target change in direction are those that undergo the largest learning modulation ( $\mathrm{Li}$ et al., 2011). If time tuning is preserved during fixation trials, it might underlie passive learning. For example, during fixation, neurons that are tuned to the direction and time of the change in the target direction would respond the most vigorously. Any inputs to these cells from other cells that are tuned to the base direction before the time of change in direction would be potentiated through spike-timing-dependent plasticity. This plasticity process should result in an increase in activity of neurons tuned to the learning direction even before the change in direction in fixation and motor trials.

Another possible learning mechanism may occur upstream from the FEF. The supplementary eye field (SEF) is a good candidate for learning the association between the movement in the base direction and the addition of a component in a learned direction (Chen and Wise, 1995; Fukushima et al., 2004). The change in SEF activity would elicit a learned response through the reciprocal connections between SEF and FEF (Huerta et al., 1987). Thus, there are several plausible sites in which observed information could be used to drive learning. Future work probing and manipulating these networks, could use the paradigm we describe here to study the implementation of motor adaptation learning in the absence of behavioral errors.

\section{Quantification of learning from fixation trials}

The learned response shown in fixation blocks (Fig. 1) can be divided into two components: the passive learning 
elicited by fixation trials and the motor learning that resulted from the test trials. The trials assessing learning are also involved in the learning process; therefore, we cannot directly measure the learning elicited exclusively by passive learning. Indirect measures suggest that most learning in fixation blocks is because of passive learning. The learned response in the first test trial, which was proceeded only by fixation trials, was similar to the learned response late in learning (Fig. $6 A$ ) and the learned response in the incongruent blocks was small (Fig. 2B).

Although we cannot completely control for the magnitude of learning from eye movement trials, we can bound the amplitude of the learned response elicited by the fixation trials. The learned response in the fixation blocks constitutes an upper bound for the amplitude of the learned response elicited by fixation trials because it contains both passive learning and a small component of motor learning. The learned response in the experiment in which the target only changed direction on fixation trials (Fig. 2E) constitutes a lower bound for learning from fixation trials. In these blocks learning was assessed using non-adaptive probe trials that reduced the learning elicited by fixation trials. We quantified these bounds by calculating the ratio of the learned response in the motor block to the learned response in the corresponding block. We estimated that passive learning in the current paradigm lay within a range of $18-48 \%$ (see Materials and Methods) of the total motor learning (learning in eye movement blocks; Fig. 1C). This estimation may not be the theoretical limit since other non-motor factors could account for the difference between passive and motor learning. For example, attention or the exact location of the stimulus on the retina at the time of the change in direction could have varied across the eye movement and fixation trials. Further research should consider the interaction between learning mechanisms elicited by motor and non-motor signals in the presence of movement. Passive learning might be elicited concurrently with mechanisms driven by motor signals or alternatively be elicited exclusively in the absence of a motor signal.

Overall, we showed that the passive observation of target motion can drive behavior characterized as motor adaptation learning. We conducted controls and explored the conditions in which passive learning is expressed. The pursuit system provides a unique model system for studying passive learning since it can be explored at the implementation level in monkeys. We suggest possible mechanisms based on the known properties of the smooth pursuit system. These hypotheses can serve as the basis for further investigations of passive motor learning in the pursuit and other systems.

\section{References}

Albus JS (1971) A theory of cerebellar function. Math Biosci 10:2561.

Bruce CJ, Goldberg ME (1985) Primate frontal eye fields. I. Single neurons discharging before saccades. J Neurophysiol 53:603-635.

Chen LL, Wise SP (1995) Neuronal activity in the supplementary eye field during acquisition of conditional oculomotor associations. $\mathrm{J}$ Neurosci 73:1101-1121.
Damasse JB, Perrinet LU, Madelain L, Montagnini A (2018) Reinforcement effects in anticipatory smooth eye movements. $J$ Vis 18:1-18.

Fukushima J, Akao T, Takeichi N, Kurkin S, Kaneko CRS, Fukushima K (2004) Pursuit-related neurons in the supplementary eye fields: discharge during pursuit and passive whole body rotation. J Neurophysiol 91:2809-2825.

Gilbert PF, Thach WT (1977) Purkinje cell activity during motor learning. Brain Res 128:309-328.

Guo CC, Ke MC, Raymond JL (2014) Cerebellar encoding of multiple candidate error cues in the service of motor learning. $\mathrm{J}$ Neurosci 34:9880-9890.

Hall NJ, Yang Y, Lisberger SG (2018) Multiple components in direction learning in smooth pursuit eye movements of monkeys. J Neurophysiol 120:2020-2035.

Held R, Freedman SJ (1963) Plasticity in human sensorimotor control. Science 142:455-462.

Herzfeld DJ, Kojima Y, Soetedjo R, Shadmehr R (2018) Encoding of error and learning to correct that error by the Purkinje cells of the cerebellum. Nat Neurosci 21:736-743.

Herzfeld DJ, Hall NJ, Tringides M, Lisberger SG (2020) Principles of operation of a cerebellar learning circuit. Elife 9:e55217.

Huerta MF, Krubitzer LA, Kaas JH (1987) Frontal eye field as defined by intracortical microstimulation in squirrel monkeys, owl monkeys, and macaque monkeys II. cortical connections. J Comp Neurol 265:332-361.

Ito M (1972) Neural design of the cerebellar motor control system. Brain Res 40:81-84.

Ito $M(1982)$ Cerebellar control of the vestibulo-ocular reflex-around the flocculus hypothesis. Annu Rev Neurosci 5:275-297.

Ito M, Kano M (1982) Long-lasting depression of parallel fiberPurkinje cell transmission induced by conjunctive stimulation of parallel fibers and climbing fibers in the cerebellar cortex. Neurosci Lett 33:253-258.

Ito M, Sakurai M, Tongroach $\mathrm{P}$ (1982) Climbing fibre induced depression of both mossy fibre responsiveness and glutamate sensitivity of cerebellar Purkinje cells. J Physiol 324:113-134.

Joshua M, Lisberger SG (2012) Reward action in the initiation of smooth pursuit eye movements. J Neurosci 32:2856-2867.

Joshua M, Lisberger SG (2015) A tale of two species: neural integration in zebrafish and monkeys. Neuroscience 296:80-91.

Krakauer JW, Mazzoni P (2011) Human sensorimotor learning: adaptation, skill, and beyond. Curr Opin Neurobiol 21:636-644.

Krauzlis RJ, Lisberger SG (1991) Visual motion commands for pursuit eye movements in the cerebellum. Science 253:568-571.

Larry N, Yarkoni M, Lixenberg A, Joshua M (2019) Cerebellar climbing fibers encode expected reward size. Elife 8:e46870.

Li JX, Medina JF, Frank LM, Lisberger SG (2011) Acquisition of neural learning in cerebellum and cerebral cortex for smooth pursuit eye movements. J Neurosci 31:12716-12726.

Lisberger SG (2010) Visual guidance of smooth-pursuit eye movements: sensation, action, and what happens in between. Neuron 66:477-491.

Liu H, Hu Y, Yang Y (2019) Cerebellar climbing fibers convey reward signals during motor learning in monkeys. Neurosci Meet Plan Chicago Soc Neurosci. Available at https://www.abstractsonline. com/pp8/\#!/7883/presentation/60524.

Lixenberg A, Joshua M (2018) Encoding of reward and decoding movement from the frontal eye field during smooth pursuit eye movements. J Neurosci 1654-1658.

Lixenberg A, Yarkoni M, Botschko Y, Joshua M (2020) Encoding of eye movements explains reward-related activity in cerebellar simple spikes. J Neurophysiol 123:786-799.

Macavoy MG, Gottlieb JP, Bruce CJ (1991) Smooth-pursuit eye movement representation in the primate frontal eye field. Cereb Cortex 1:95-102.

Mazzoni P, Krakauer JW (2006) An implicit plan overrides an explicit strategy during visuomotor adaptation. J Neurosci 26:3642-3645. 
McDougle SD, Ivry RB, Taylor JA (2016) Taking aim at the cognitive side of learning in sensorimotor adaptation tasks. Trends Cogn Sci 20:535-544.

Medina JF, Lisberger SG (2008) Links from complex spikes to local plasticity and motor learning in the cerebellum of awake-behaving monkeys. Nat Neurosci 11:1185-1192.

Medina JF, Carey MR, Lisberger SG (2005) The representation of time for motor learning. Neuron 45:157-167.

Mostafa AA, 't Hart BM, Henriques DYP (2019) Motor learning without moving: proprioceptive and predictive hand localization after passive visuoproprioceptive discrepancy training. PLoS One 14: e0221861.

Rashbass C, Westheimer G (1961) Independence of conjugate and disjunctive eye movements. J Physiol 159:361-364.

Schafer RJ, Moore T (2011) Selective attention from voluntary control of neurons in prefrontal cortex. Science 332:1568-1571.

Schall JD, Hanes DP, Thompson KG, King DJ (1995) Saccade target selection in frontal eye field of macaque. I. Visual and premovement activation. J Neurosci 15:6905-6918.

Schoppik D, Nagel KI, Lisberger SG (2008) Cortical mechanisms of smooth eye movements revealed by dynamic covariations of neural and behavioral responses. Neuron 58:248-260.
Shadmehr R, Smith MA, Krakauer JW (2010) Error correction, sensory prediction, and adaptation in motor control. Annu Rev Neurosci 33:89-108.

Sommer MA, Wurtz RH (2006) Influence of the thalamus on spatial visual processing in frontal cortex. Nature 444:374-377.

Stone LS, Lisberger SG (1990) Visual responses of Purkinje cells in the cerebellar flocculus during smooth-pursuit eye movements in monkeys. II. Complex spikes. J Neurophysiol 63:1262-1275.

Suvrathan A, Payne HL, Raymond JL (2016) Timing rules for synaptic plasticity matched to behavioral function. Neuron 92:959-967.

Voogd J, Schraa-Tam CKL, Van Der Geest JN, De Zeeuw Cl (2012) Visuomotor cerebellum in human and nonhuman primates. Cerebellum 11:392-410.

Wolpert DM, Miall RC (1996) Forward models for physiological motor control. Neural Netw 9:1265-1279.

Wolpert DM, Miall RC, Kawato M (1998) Internal models in the cerebellum. Trends Cogn Sci 2:338-346.

Yang Y, Lisberger SG (2010) Learning on multiple timescales in smooth pursuit eye movements. J Neurophysiol 104:2850-2862.

Yang Y, Lisberger SG (2014) Purkinje-cell plasticity and cerebellar motor learning are graded by complex-spike duration. Nature 510:529-532. 